\title{
1 Animal, fungi, and plant genome sequences 2 harbour different non-canonical splice sites
}

4 Katharina Frey ${ }^{1}$ and Boas Pucker ${ }^{1, *}$

5

$6{ }^{1}$ Genetics and Genomics of Plants, Center for Biotechnology (CeBiTec), Bielefeld

7 University, Bielefeld, Germany

8 "Corresponding author

11 Email addresses:

12 KF: katharina.frey@uni-bielefeld.de

13 BP: bpucker@cebitec.uni-bielefeld.de

ORCIDs:

16 


\section{Abstract}

32 Most protein encoding genes in eukaryotes contain introns which are interwoven with exons. After transcription, introns need to be removed in order to generate the final mRNA which can be translated into an amino acid sequence. Precise excision of introns by the spliceosome requires conserved dinucleotides which mark the splice sites. However, there are variations of the highly conserved combination of GT at the $5^{\prime}$ end and $A G$ at the 3 ' end of an intron in the genome. GC-AG and AT-AC are two major non-canonical splice site combinations which have been known for years. During the last years, various minor non-canonical splice site combinations were detected with numerous dinucleotide permutations. Here we expand systematic investigations of non-canonical splice site combinations in plants to all eukaryotes by analysing fungal and animal genome sequences. Comparisons of splice site combinations between these three kingdoms revealed several differences such as a substantially increased CT-AC frequency in fungal genome sequences. Canonical GT-AG splice site combinations in antisense transcripts could be one explanation for this observation. In addition, high numbers of GA-AG splice site combinations were observed in Eurytemora affinis and Oikopleura dioica. A variant in one U1 snRNA isoform might allow the recognition of GA as 5' splice site. In depth investigation of splice site usage based on RNA-Seq read mappings indicates a generally higher flexibility of the 3 ' splice site compared to the 5' splice site across animals, fungi, and plants.

\section{Introduction}

54 Splicing, the removal of introns after transcription, is an essential step during the 55 generation of mature mRNAs in eukaryotes. This process allows variation which provides the basis for quick adaptation to changing conditions ${ }^{1,2}$. Alternative splicing,

57 e.g. skipping exons, usage of alternative 5' or 3' splice sites and the retention of 58 introns, results in an enormous diversity of synthesized proteins and therefore 59 substantially expands the diversity of products encoded in eukaryotic genomes ${ }^{3-6}$.

60 The full range of functions as well as the evolutionary relevance of introns are still 61 under discussion ${ }^{7}$. However, introns are energetically expensive for the cell to 62 maintain as the transcription of introns costs time and energy and the removal of 
63 introns has to be exactly regulated ${ }^{8}$. Dinucleotides at both intron/exon borders mark 64 the splice sites and are therefore highly conserved ${ }^{9}$. GT at the $5^{\prime}$ end and AG at the 653 ' end of an intron form the canonical splice site combination on DNA level. More 66 complexity arises through non-canonical splice site combinations, which deviate from 67 the highly conserved canonical one. Besides the major non-canonical splice site 68 combinations GC-AG and AT-AC, several minor non-canonical splice site 69 combinations have been detected before ${ }^{9,10}$.

70 Furthermore, the position of introns in homologous genes across organisms, which 71 diverged 500-1500 million years ago, are not conserved ${ }^{11}$. In addition, many intron 72 sequences mutate at a higher rate due to having much less of an impact on the 73 reproductive fitness of an organism compared to a mutation located within an exon 74 12. These factors, along with the existence of several non-canonical splice sites, 75 make the complete prediction of introns, even in non-complex organisms like yeast, 76 almost impossible 13,14. Moreover, most introns which can be predicted 77 computationally still lack experimental support ${ }^{15}$.

78 Splice sites are recognised during the splicing process by a complex of snRNAs and 79 proteins, the spliceosome ${ }^{16}$. U2-spliceosome and U12-spliceosome are two 80 subtypes of this complex which comprise slightly different proteins with equivalent 81 functions ${ }^{17-19}$. Although the terminal dinucleotides are important for the splicing 82 process, these splice sites are not sufficient to determine which spliceosome is 83 processing the enclosed intron ${ }^{20}$. This demonstrates the complexity of the splicing 84 process which involves additional signals present in the DNA. Even though multiple 85 mechanisms could explain the splicing process, the exact mechanism of non86 canonical splicing is still not completely resolved ${ }^{5}$.

87 Branching reaction and exon ligation are the two major steps of splicing ${ }^{21,22}$. In the 88 branching reaction, the 2'-hydroxyl group of the branchpoint adenosine initiates an 89 attack on the $5^{\prime}$-phosphate of the donor splice site ${ }^{23,24}$. This process leads to the 90 formation of a lariat structure. Next, the exons are ligated and the intron is released 91 through activity of the 3'-hydroxyl group of the $5^{\prime}$ exon at the acceptor splice site ${ }^{21}$.

92 Previous in-depth analyses of non-canonical splice sites in fungi and animals were 93 often focused on a single or a small number of species ${ }^{9,25,26}$. Several studies 94 focused on canonical GT-AG splice sites but neglected non-canonical splice sites 
27,28 . Our understanding of splice site combinations is more developed in plants compared to other kingdoms ${ }^{10,29-33}$. Previous works reported $98 \%$ GT-AG splice site combinations in fungi ${ }^{25}, 98.7 \%$ in plants, ${ }^{10}$ and $98.71 \%$ in animals ${ }^{9}$. Consequently, the proportion of non-canonical splice sites, other than the canonical splice site GT$A G$, is around or below $2 \%^{9,10,25}$. To the best of our knowledge, it is not known if the value reported for mammals is representative for all animals. Non-canonical splice site combinations can be divided into major non-canonical GC-AG and AT-AC combinations and the minor non-canonical splice sites which are all other dinucleotide combinations at the terminal intron positions. The combined frequency of all minor non-canonical splice site combinations is low e.g. $0.09 \%$ in plants, but still exceeds the frequency of the major non-canonical AT-AC splice sites ${ }^{10}$. Despite this apparently low frequency, non-canonical splice site combinations have a substantial impact on gene products, especially on exon-rich genes ${ }^{10}$. Over $40 \%$ of plant genes with exactly 40 exons are affected ${ }^{10}$.

Consideration of non-canonical splice sites is important for gene prediction approaches, because $a b$ initio identification of these splice sites is computationally extremely expensive and therefore rarely applied ${ }^{29}$. Moreover, as many human pathogenic mutations occur at the donor splice site ${ }^{34}$, it is of great interest to understand the occurrence and usage of non-canonical splice sites. Therefore, several non-canonical splice sites containing $A G$ at the acceptor site were investigated in human fibroblasts ${ }^{34}$. Alongside this, fungi are interesting due to pathogenic properties and importance in the food industry ${ }^{35}$. Since splicing leads to high protein diversity ${ }^{3-6}$, the analysis of splicing in fungi is important with respect to biotechnological applications e.g. development of new products.

Non-canonical splice sites are frequently considered as artifacts ${ }^{36}$ and therefore excluded from analyses ${ }^{27,28}$. Further, RNA editing of GT-AA to GT-AG splice sites on RNA level is possible ${ }^{37}$. This leads to the transformation of non-canonical splice site combinations into canonical ones. Previous studies supported minor non-canonical splice site combinations in single or few species ${ }^{9,25,26}$ and systematically across plants ${ }^{10,29-33}$. In this study, a collection of annotated genome sequences from 130 fungi and 489 animal species was screened for canonical and non-canonical splice site combinations in representative transcripts. RNA-Seq data sets were harnessed to identify biologically relevant and actually used splice sites based on the available 
128 annotation. Non-canonical splice site combinations, which appeared at substantially 129 higher frequency in a certain kingdom or species, were analysed in detail. As 130 knowledge about splice sites in plants was available from previous investigations $1311^{10,29}$, a comparison between splice sites in fungi, animals and plants was performed.

\section{Results and Discussion}

\section{Analysis of non-canonical splice sites}

135 In total, 64,756,412 (Supplementary Data S1) and 2,302,340 (Supplementary Data 136 S2) splice site combinations in animals and fungi, respectively, were investigated 137 based on annotated genome sequences (Supplementary Data S3 and 138 Supplementary Data S4). The average frequency of the canonical splice site 139 combination GT-AG is $98.3 \%$ in animals and $98.7 \%$ in fungi, respectively. These 140 values exceed the $97.9 \%$ previously reported for plants ${ }^{10}$, thus indicating a generally

141 higher frequency of non-canonical splice site combinations in plants. As previously 142 speculated ${ }^{10}$, a generally less accurate splicing system in plants could be an 143 adaptation to changing environments through the generation of a larger transcript 144 diversity. Since most plants are not able to change their geographic location, the 145 tolerance for unfavorable conditions should be stronger than in animals. The lower 146 proportion of non-canonical splice site combinations in fungi compared to animals 147 seems to contradict this hypothesis. However, the genome size and complexity 148 needs to be taken into account here. Based on the available assemblies, the average 149 animal genome is significantly larger than the average fungal genome (Mann150 Whitney U-Test; $p=5.64 e-68$ ) (Supplementary Data S5). Although the average animal 151 genome sequence (median=998 Mbp) is longer than the plant average (median=467 $152 \mathrm{Mbp})$, plant genome sequences harbour more non-canonical splice sites 153 (Supplementary Data S6, Supplementary Data S7 ${ }^{10}$ ).

154 Average percentages of the most important splice site combinations were 155 summarized per kingdom and over all analysed genomes (Table 1). The number of 156 canonical and non-canonical splice site combinations per species was also 157 summarized (Supplementary Data S8 and Supplementary Data S9). A higher 158 percentage of non-canonical splice sites was observed in animals in comparison to 159 fungi. Several species strongly exceeded the average values for major and minor 
160 non-canonical splice sites. The fungal species Meyerozyma guilliermondi shows 161 approximately $6.67 \%$ major and $13.33 \%$ minor non-canonical splice sites.

162 Eurytemora affinis (copepod) and Oikopleura dioica (tunicate) reveal approximately

$16310 \%$ minor non-canonical splice sites. In summary, the observed frequencies of

164 canonical and major non-canonical splice site combinations are similar to the pattern 165 previously reported for plants ${ }^{10}$, but some essential differences and exceptions were 166 found in animals and fungi. Previous studies already revealed that non-canonical 167 splice site combinations are not just the result of sequencing errors ${ }^{9,10,29,38}$. Here, we 168 investigated the position of sequence variants in plants, fungi, and animals with 169 respect to splice sites. The average frequency of sequence variants at splice sites is 170 far below 1\% (Supplementary Data S10). Although non-canonical splice sites are 171 generally more likely to harbour sequence variants than canonical ones, these 172 sequence variants can only account for a very small proportion of non-canonical 173 splice site combinations.

175 Table 1: Splice site combination frequencies in animals, fungi, and plants. Only the 176 most frequent combinations are displayed here and all minor non-canonical splice site 177 combinations are summarized as one group ("others"). A full list of all splice site 178 combinations is available (Supplementary Data S1 and Supplementary Data S2).

\begin{tabular}{lllll}
\hline & GT-AG & GC-AG & AT-AC & others \\
\hline animals & $98.334 \%$ & $0.983 \%$ & $0.106 \%$ & $0.577 \%$ \\
fungi & $98.715 \%$ & $1.009 \%$ & $0.019 \%$ & $0.257 \%$ \\
plants & $97.886 \%$ & $1.488 \%$ & $0.092 \%$ & $0.534 \%$ \\
all & $98.265 \%$ & $1.074 \%$ & $0.101 \%$ & $0.560 \%$ \\
\hline
\end{tabular}

179

180 Different properties of the genome sequences of all investigated species were 181 analysed to identify potential explanations for the splice site differences 182 (Supplementary Data S6 and Supplementary Data S7). In fungi, the average number 183 of introns per gene is 1.49 and the average GC content is $47.1 \%$ ( \pm 7.39 ; s.d.). In 184 animals, each gene contains on average 6.95 introns and the average GC content is $18539.4 \%$ ( \pm 3.87 ; s.d.). The average number of introns per gene in plants is 4.15 and 186 the average GC content $36.3 \%$ ( \pm 8.84 ; s.d.). This difference in the GC content 187 between fungi and animals/plants could be associated with the much lower frequency 
188 of AT-AC splice site combinations and the higher frequency of CT-AC splice site 189 combinations in fungi (Figure 1). CT-AC has a higher GC content than the AT rich 190 AT-AC splice site combination. A generally higher GC content could result in the 191 higher GC content within splice site combinations due to the overall mutation rates in 192 these species.

194 Figure 1: Frequencies of non-canonical splice site combinations in animals, fungi, and 195 plants. The frequency of non-canonical splice site combinations across the 489 animal (red), 196130 fungal (blue) and 121 plant (green) genomes is shown. Normalization of the absolute 197 number of each splice site combination was performed per species based on the total 198 number of splice sites. The frequency of the respective splice site combination of each 199 species is shown on the left-hand side and the percentage of the respective splice site 200 combination on top of each box plot. The dashed line represents the mean frequency of the 201 respective splice site combination over all investigated species. The box plots are ordered 202 (from left to right) according to the mean frequency.

203

204 A comparison of the genome-wide GC content to the GC content of all splice sites 205 revealed a weak correlation in the analysed fungi $(r \approx 0.236, p \approx 0.008)$. Species with a 206 high genomic GC content tend to show a high GC content in the splice site 207 combinations in the respective species. A similar correlation was found in plant $208(r \approx 0.403, p \approx 4.505 e-06)$ and animal species $(r \approx 0.434, p \approx 7.866 e-24)$ (Supplementary 209 Data S11). Additionally, the GC content in fungal genomes is substantially exceeding 210 the average GC content of plant and animal genomes. Since genomic GC content 211 and intronic GC content strongly correlate (animals: $r \approx 0.968, p \approx 2.357 e-292$, plants: $212 r \approx 0.974, p \approx 8.987 e-79$ and fungi: $r \approx 0.950, p \approx 2.800 e-66)$, the results obtained in the 213 analysis above are representative for both methods of GC content calculation 214 (Supplementary Data S11). Since splicing of U12 introns, which often show the major 215 non-canonical AT-AC splice site combination, requires the presence of the minor U12 216 spliceosome, we screened the genome sequences of all investigated species for 217 components of this spliceosome. As differences in the genome sequence 218 completeness and continuity as well as sequence divergent from bait sequences can 219 impact the results, we only state the presence of the U12 spliceosome in some 220 species while the absence in the remaining species cannot be demonstrated. The 
221 comparison of annotated AT-AC splice site combinations between species with and 222 without the minor U12 spliceosome revealed significantly higher numbers of this 223 major non-canonical splice site combination in species with U12 spliceosomes 224 (Mann-Whitney U-Test: $p \approx 3.8 \mathrm{e}-12$ (plants) and $\mathrm{p} \approx 1.8 \mathrm{e}-15$ (animals)). Although many 225 fungi are known to have a minor U12 spliceosome ${ }^{19}$ we only detected corresponding 226 RNA genes in one species (Cutaneotrichosporon oleaginosum) and thus refrained 227 from any conclusions about the situation in fungi.

228 The most frequent non-canonical splice site combinations show differences between 229 animals, fungi, and plants (Figure 1). In fungal genome sequences, the splice site 230 CT-AC is substantially more frequent than the splice site combination AT-AC. 231 Regarding the splice site combination GA-AG in animal genome sequences, two 232 outliers are clearly visible: $E$. affinis and $O$. dioica show more GA-AG splice site 233 combinations than GC-AG splice site combinations.

234 Despite overall similarity in the pattern of non-canonical splice site combinations 235 between kingdoms, specific minor non-canonical splice site combinations were 236 identified at much higher frequency in some fungal and animal species. First, RNA237 Seq data was harnessed to validate these unexpected splice site combinations. Next, 238 the frequencies of selected splice site combinations across all species of the 239 respective kingdom were calculated. The correlation between the size of the 240 incorporated RNA-Seq data sets and the number of supported splice sites was 241 examined as well (Supplementary Data S12). In animals, there is a correlation $242(r \approx 0.417, p \approx 0.022)$ between number of supported splice sites and total number of 243 sequenced nucleotides in RNA-Seq data. For fungi, no correlation between number 244 of supported splice sites and size of the RNA-Seq data sets could be observed. It is 245 important to note that the number of available RNA-Seq data sets from fungi was 246 substantially lower.

247 Further, analysis of introns with canonical and non-canonical splice site 248 combinations, respectively, revealed that a higher number of introns is associated 249 with a higher proportion of non-canonical splice sites (Supplementary Data S13).

250

251 High diversity of non-canonical splice sites in animals 
252 Kupfer et al. suggested that splicing may differ between fungi and vertebrates ${ }^{25}$. Our

253 results indicate substantial differences in the diversity of splice site combinations

254 other than GT-AG and GC-AG in fungi $\left(H^{\prime} \approx 0.0277\right)$ and animals $\left(H^{\prime} \approx 0.0637\right)$

255 (Kruskal-Wallis: $p \approx 0$ ). Besides the overall high proportion of minor non-canonical

256 splice sites (Table 1), differences between species are high (Figure 1). The slightly

257 higher interquartile range of splice site combination frequencies in animal species

258 and especially in plant species (Figure 1A and $\mathrm{C}$ ), together with the relatively high

259 frequency of "other" splice sites in animals and plants (Table 1) suggest more

260 variation of splice sites in the kingdoms of animals and plants compared to the

261 investigated fungal species. Thus, the high diversity of splice sites could be

262 associated with the higher complexity of animal and plant genomes. In addition, the

263 difference in prevalence between the major non-canonical splice site combination

264 GC-AG and minor non-canonical splice site combinations is smaller in animals

265 compared to fungi and plants (Figure 1).

266 GA-AG is a frequent non-canonical splice site combination in some animal species.

267 Two species, namely $E$. affinis and $O$. dioica, showed a much higher abundance of

268 GA-AG splice site combinations compared to the other investigated species (Figure

269 1A). RNA-Seq reads support 5,795 (22,866 \% (average)) of all GA-AG splice site

270 combinations of both species. GA-AG splice sites are supported in all analysed

271 species with a slightly lower frequency of $19.032 \%$. In $E$. affinis and $O$. dioica, the

272 number of the GA-AG splice site combination exceeds the number of the major non-

273 canonical splice site combination GC-AG.

274 For $E$. affinis, the high frequency of the GA-AG splice site combinations was 275 described previously when GA-AG was detected in 36 introns ${ }^{39}$. We quantified the

276 proportion of GA-AG splice site combinations to $3.2 \%(5,345)$ of all 166,392

277 supported splice site combinations in this species. The donor splice site GA is 278 flanked by highly conserved upstream AG and a downstream A (Figure 2). Both 279 species, E. affinis (Figure 2A,B) and O. dioica (Figure 2C,D), show striking similarities 280 at several positions of donor and acceptor splice sites in addition to the GA-AG 281 dinucleotides. As the arthropod E. affinis ${ }^{40}$ and the chordate $O$. dioica ${ }^{41}$ belong to 282 different phyla, the conservation of sequences flanking the donor and acceptor splice 283 sites and the ability to splice GA-AG introns might be explained by convergent 
284 evolution or rather an ancestral trait which was only kept in a few species, including

285 E. affinis and O. dioica.

286

287

Figure 2: Flanking positions of GA-AG splice site combinations in Eurytemora affinis

288

(a,b) and Oikopleura dioica (c,d). All splice site combinations (a,c) as well as all 5,795 with

289 RNA-Seq data supported splice site combinations (b,d) of these two species were investigated. Seven exonic and seven intronic positions are displayed at the donor and

291 acceptor splice sites. Underlined bases represent the terminal dinucleotides of the intron i.e. the donor and acceptor splice site.

293

294 Possible mechanisms which could explain these GA-AG splice site combinations are 295 RNA editing or template switching by a reverse transcriptase. A high GC content of 296 non-canonical splice site combinations, which is not valid for GA-AG splice sites, 297 could facilitate the formation of secondary structures ultimately leading to template 298 switching ${ }^{36}$. However, RNA editing can lead to the formation of canonical splice sites 299 on RNA level even though a non-canonical splice site is present on DNA level ${ }^{37}$.

300

301 Figure 3: Hypothetical binding of the U1 snRNA to the pre-mRNA. A) Binding sequence 302 of the canonical U1 snRNA to the canonical 5' splice site GU (GT on DNA). B) Hypothetical binding sequence of the non-canonical U1 snRNA $(C>T)$ to the non-canonical 5 ' splice site 304 GA.

305 Efficient splicing of the splice site combination GA-AG was detected in human fibroblast growth factor receptor genes ${ }^{42}$. Further, it was suggested that this splicing event is, among other sequence properties, dependent on a canonical splice site six nucleotides upstream ${ }^{42}$, which does not exist in the genome sequence of the species investigated here (Figure 2). An analysis of all five potential U1 snRNAs in E. affinis did reveal one single nucleotide polymorphism in the binding site of the 5 ' splice site

311 from $\mathrm{C}$ to $\mathrm{U}$ at position eight in one of these $U 1$ snRNAs. This could result in the 312 binding of pre-mRNAs originating from A/GATAAGT instead of AG/GTAAGT (Figure 313 3) ${ }^{39,43}$. Although this would imply an elegant way for the splicing of GA-AG splice 314 sites, the same variation was also detected in putative human U1 snRNAs. 315 Therefore, another mechanism or additional factor might be causal for splicing of 
316 introns containing the GA-AG splice site combination. A modified copy of 317 spliceosomal components is a likely explanation for the observed GA-AG splice site 318 combination. However, no higher amplification ratio of spliceosome parts was 319 observed in E. affinis and O. dioica compared to other animal species.

\section{CT-AC is a frequent splice site combination in fungal annotations}

322 Although the general frequency pattern of fungal splice site combinations is similar to 323 plants and animals, several fungal species displayed a high frequency of minor non324 canonical CT-AC splice site combinations. This co-occurs with a lower frequency of 325 AT-AC splice site combinations. Non-canonical splice sites in fungi were, so far, only 326 described in studies which focussed on a single or a few species. An analysis in the 327 fungus-like microorganism Phytophthora sojae ${ }^{44,45}$, revealed $3.4 \%$ non-canonical 328 splice site combinations GC-AG and CT-AC ${ }^{46}$. Our findings indicate, that the minor 329 non-canonical splice site combination CT-AC occurs with a significantly (Mann330 Whitney $U$-Test; $p \approx 0.00035$ ) higher frequency in the annotation of fungal genome 331 sequences than the major non-canonical splice site combination AT-AC. In contrast, 332 the frequency of AT-AC in animals $(p \approx 9.560 \mathrm{e}-10)$ and plants $(p \approx 5.464 \mathrm{e}-24)$ exceeds 333 the CT-AC frequency significantly (Figure 4A). For the splice site combination CT-AC 334 a sequence logo, which shows the conservation of this splice site in four selected 335 species, was designed (Figure 4B). In summary, we conclude that CT-AC is a major 336 non-canonical splice site combination in fungi, while AT-AC is not. The highest 337 frequencies of the splice site combination CT-AC, supported by RNA-Seq reads, 338 were observed in Alternaria alternata, Aspergillus brasiliensis, Fomitopsis pinicola, 339 and Zymoseptoria tritici (approx. 0.08 - $0.09 \%$ ). As AT-AC was described as major 340 non-canonical splice site, these findings might indicate a different splice site pattern 341 in fungi compared to animals and plants (Figure 4).

343 Figure 4: CT-AC frequency exceeds AT-AC frequency in the annotation of fungal 344 genome sequences. A) Number of the minor non-canonical splice site combination CT-AC 345 in comparison to the major non-canonical splice site combination AT-AC in each kingdom 346 (Mann-Whitney U-Test; fungi: $p \approx 0.00035$, animals: $p \approx 9.560 \mathrm{e}-10$, plants: $p \approx 5.464 \mathrm{e}-24$ ). The 347 dashed line represents the mean frequency of the respective splice site combination over all 348 investigated species. B) Sequence logo for the splice site combination CT-AC in four 
349

350

351

352

353

354

355

356

357

358

359

360

361

362

363

364

365

366

367

368

369

370

371

372

373

374

375

376

selected fungal species (Alternaria alternata, Aspergillus brasiliensis, Fomitopsis pinicola and Zymoseptoria triticl). In total, 67 supported splice sites with this combination were used to generate the sequence logo.

The presence of antisense transcripts which would be spliced at a canonical GT-AG splice site combination (reverse complement of CT-AC) is a likely explanation. At least, stranded RNA-Seq data sets are required to investigate this hypothesis in fungi by differentiating between transcripts of both strands. Frequently encountered artifacts caused by reverse transcription ${ }^{36}$ might be avoided in the future through direct RNA sequencing ${ }^{47}$. Due to the very limited availability of suitable data sets for fungal species we have to leave this question for future studies.

\section{Intron size analysis}

Assuming that non-canonical splice sites are not used or used at a lower efficiency, it could be assumed that introns are more often retained than introns with canonical splice sites. A possible consequence of intron retention could be frameshifts unless the intron length is a multiple of three. Therefore, we investigated a total of $8,060,924,737,783$ and 2,785,484 transcripts across animals, fungi, and plants, respectively, with respect to their intron lengths. Introns with a length divisible by three could be kept in the final transcript without causing a shift in the reading frame, because they add complete codons to the transcript. There is no significant difference between introns with different splice site combinations (Table 2). The ratio of introns with a length divisible by 3 is very close to $33.3 \%$ which would be expected based on an equal distribution. The only exception are minor non-canonical splice site combinations in fungi which are slightly less likely to occur in introns with a length divisible by 3 .

Table 2: Proportion of introns with length divisible by 3 . The results of intron length analysis for selected splice site combinations for animals, fungi and plants are shown.

\begin{tabular}{llll}
\hline & splice site combination & introns divisible by 3 & number of introns divisible by 3 \\
\hline \multirow{3}{*}{ animals } & GT-AG & $33.39 \%$ & $\mathrm{n}=63677347$ \\
& AT-AC & $32.51 \%$ & $\mathrm{n}=68919$ \\
& GC-AG & $33.04 \%$ & $\mathrm{n}=636823$
\end{tabular}




\begin{tabular}{|c|c|c|c|}
\hline & others & $32.76 \%$ & $\mathrm{n}=496411$ \\
\hline & GT-AG & $33.93 \%$ & $n=2273756$ \\
\hline \multirow[t]{4}{*}{ fungi } & AT-AC & $33.18 \%$ & $n=428$ \\
\hline & GC-AG & $33.36 \%$ & $\mathrm{n}=23224$ \\
\hline & others & $31.25 \%$ & $\mathrm{n}=6240$ \\
\hline & GT-AG & $33.3 \%$ & $\mathrm{n}=14227286$ \\
\hline \multirow[t]{3}{*}{ plants } & AT-AC & $32.62 \%$ & $\mathrm{n}=13411$ \\
\hline & GC-AG & $32.93 \%$ & $n=216326$ \\
\hline & others & $32.4 \%$ & $n=93638$ \\
\hline
\end{tabular}

378 Conservation of non-canonical splice site combinations across species

379 In total, $A$. thaliana transcripts containing 1,073 GC-AG, 64 AT-AC and 19 minor non-

380 canonical splice sites were aligned to transcripts of all plant species. Homologous 381 intron positions were checked for non-canonical splice sites. GC-AG splice site 382 combinations were conserved in 9,830 sequences, matched with other non-canonical 383 splice site combinations in 121 cases, and aligned to GT-AG in 13,045 sequences. 384 Given that the dominance of GT-AG splice sites was around $98 \%$, the number 385 observed here indicates a strong conservation of GC-AG splice site combinations. 386 AT-AC splice site combinations were conserved in 967 other sequences, matched 387 with other non-canonical splice site combinations in 93 cases, and aligned to GT-AG 388 in 157 sequences. These numbers indicate a conservation of AT-AC splice site 389 combinations, which exceeds the conservation of GC-AG splice site combinations 390 substantially. Minor non-canonical splice sites were conserved in 48 other 391 sequences, matched with other non-canonical splice site combinations in 64 cases, 392 and were aligned to a canonical GT-AG splice site in 213 cases. This pattern 393 suggests that most non-canonical splice site combinations are either $(A)$ mutations of 394 the canonical ones or (B) mutated towards GT-AG splice site combinations.

395 The power of this analysis is currently limited by the quality of the alignment. 396 Although splice site combinations should be aligned properly in most cases, small 397 differences in the number could be caused by ambiguous situations. It is likely that 398 both events stated above account for a fraction of splice site combinations. To assign 399 each non-canonical splice site combination to A or B, a tool for automatic inspection 400 of the observed phylogenetic pattern would be required. To assess the feasibility of 401 this approach, we investigated the conservation of non-canonical splice sites in 402 transcripts of Armillaria gallica as this species shows a high number of non-canonical 
403 splice sites in the annotation and the set of fungal genome sequences has a feasible 404 size for this analysis. After identification of putative homologous sequences in other 405 species, phylogenetic trees of these sequences were inspected. Transcripts with 406 non-canonical splice site combinations are clustered in clades which also harbour 407 transcripts without non-canonical splice site combinations. We analysed trees of all 408 transcripts which have similar transcripts with non-canonical splice site combinations 409 in at least 10 other species and observed on average 5 transcripts with a non410 canonical splice site combination among the 10 closest relatives. This number is 411 exceeding the expectation based on the overall frequency of less than $3 \%$ non412 canonical splice site combinations, thus indicating conservation of non-canonical 413 splice sites. Due to this apparently complex evolutionary pattern, we do not know if 414 these clades originated from a non-canonical splice site combination which was 415 turned into a canonical one multiple times $(B)$ or if a non-canonical splice site 416 combination evolved multiple times $(A)$.

\section{Usage of non-canonical splice sites}

419 Non-canonical splice site combinations were described to have regulatory roles by 420 slowing down the splicing process ${ }^{48}$. Previous reports also indicated that non421 canonical splice site combinations might appear in pseudogenes ${ }^{9,10}$. To analyse a 422 possible correlation of non-canonical splice sites with low transcriptional activity, we 423 compared the transcript abundance of genes with non-canonical splice site 424 combinations to genes with only canonical GT-AG splice site combinations (Figure $4255 \mathrm{~A})$. Genes with at least one non-canonical splice site combination are generally less 426 likely to be lowly expressed than genes with only canonical splice sites. While this 427 trend holds true for all analysed non-canonical splice site combination groups, GC428 AG and AT-AC containing genes display especially low proportions of genes with low 429 FPKMs. We speculate that a stronger transcriptional activity of genes with non430 canonical splice sites compensates for lower turnover rates in the splicing process. 431 The regulation of these genes might be shifted from the transcriptional to the post432 transcriptional level. This trend is similar for animals and plants (Supplementary Data 433 S14). In fungi, genes with minor non-canonical splice sites display relatively high 434 proportions of genes with low FPKMs. Moreover, a higher number of non-canonical 
435 splice sites per gene is associated with a lower expression. This leads to the 436 suggestion, that non-canonical splice sites occur more often within pseudogenes.

438 Figure 5: Usage of non-canonical splice sites in plant species. A) Comparison of the 439 transcript abundance (FPKMs) of genes with non-canonical splice site combinations to genes 440 with only canonical GT-AG splice site combinations. GC-AG and AT-AC containing genes 441 display especially low proportions of genes with low FPKMs. This leads to a higher transcript 442 abundance of genes with low FPKMs. B) Comparison of the usage of 5 ' and 3 ' splice sites. 443 On the x-axis, the difference between the 5 ' splice site usage and the usage of the 3 ' splice 444 site is shown. A fast drop of values when going to the negative side of the $x$-axis indicates 445 that the 3 ' splice site is probably more flexible than the $5^{\prime}$ splice site.

447 Introns are mostly defined by phylogenetically conserved splice sites, but 448 nevertheless some variation of these splice sites is possible ${ }^{9,10,25,26,46}$. To understand 449 the amount of flexibility in respect to different terminal dinucleotides, we compared 450 the usage of donor and acceptor splice sites over 4,141,196 introns in plants, $4513,915,559$ introns in animals, and 340,619 introns in fungi (Figure 5B). The plot 452 shows that the 3 ' splice site seems to be more flexible than the 5' splice site which 453 was observed in all three kingdoms. Our observations align well with previous 454 findings of a higher flexibility at the $3^{\prime}$ splice site compared to the $5^{\prime}$ splice site. A 455 mutated 5' splice site represses the removal of the upstream intron ${ }^{10,49,50}$. Further, 456 for plants and animals, the difference between the usage of the 5' splice site and the 4573 ' splice site is notably higher for introns with the splice site combination GC-AG.

458 One important limitation of this investigation is the sparse availability of stranded 459 RNA-Seq data sets and direct RNA sequencing data sets. Therefore, it is not 460 possible to rule out the involvement of antisense transcripts, which have been 461 observed before ${ }^{51}$. In three cases, these antisense transcripts could be spliced at a 462 canonical or major non-canonical splice site combination, while appearing as a minor 463 non-canonical splice site on the investigated strand. Previous reports of antisense 464 transcripts ${ }^{52}$ suggest an explanation for this observation. Although bona fide non465 canonical splice site combinations are present in many plant transcripts ${ }^{10}$, additional 466 transcript isoforms might exist. To evaluate the relevance of such alternative 467 isoforms, we assessed the contribution of isoforms to the overall abundance of 
468 transcripts of a gene. Therefore, the usage of splice sites flanking an intron was

469 compared to the average usage of splice sites. This reveals how often a certain

470 intron is removed by splicing. Introns with low usage values might only be involved in

471 minor transcript isoforms. While most introns display no or very small differences,

472 GT-AG introns deviate from this trend. This indicates that non-canonical splice site

473 combinations are frequently part of the dominant isoform. Again, these findings were

474 similar for all of the investigated kingdoms.

475

476 Our investigation of non-canonical splice sites in animal, fungal and plant genome 477 sequences revealed kingdom specific differences. Animal species with a high 478 proportion of annotated GA-AG splice site combinations were examined. Further, 479 properties of introns and splice sites were analysed. One aspect of this analysis is, 480 that the $3^{\prime}$ splice site seems to be more flexible than the 5' splice site, which was 481 observed in all three kingdoms. Across fungal genome sequences, the splice site 482 combination CT-AC is more frequent than the splice site combination AT-AC. This 483 makes CT-AC a major non-canonical splice site combination in fungal species, while 484 AT-AC should be considered a minor non-canonical splice site in fungi. Overall, our 485 findings demonstrate the importance of considering non-canonical splice sites 486 despite their low relative frequency in comparison to the canonical splice site 487 combination GT-AG. RNA-Seq data supported the existence and usage of numerous 488 non-canonical splice site combinations. By neglecting non-canonical splice sites, 489 bona fide genes might be excluded or at least structurally altered.

490

491 Methods

492 Analysis and validation of splice site combinations

493 A detailed technical description of all included scripts with usage examples is 494 available from the corresponding github repository 495 (https://github.com/bpucker/ncss2018). A wrapper script is included to automatically 496 perform the analyses based on provided genome sequence (FASTA), annotation 497 (GFF3), and RNA-Seq read mapping (BAM) per species of interest. 
498 Genome sequences (FASTA) and corresponding annotations (GFF3) of 130 fungal 499 species and 489 animal species were retrieved from the NCBI. Representative 500 transcript and peptide sequences were extracted as described before ${ }^{10}$. General 501 statistics were calculated using a Python script ${ }^{10}$. The completeness of all data sets 502 was assessed with BUSCO v3 ${ }^{53}$ running in protein mode on the representative 503 peptide sequences using the reference data sets 'fungi odb9' and 'metazoa odb9', 504 respectively ${ }^{54}$ (Supplementary Data S15 and Supplementary Data S16).

505 To validate the detected splice site combinations, paired-end RNA-Seq data sets 506 were retrieved from the Sequence Read Archive ${ }^{55}$ (Supplementary Data S17 and 507 Supplementary Data S18). The following validation approach ${ }^{10}$ utilized STAR 508 v2.5.1b ${ }^{56}$ for the read mapping and Python scripts for downstream processing 509 (https://doi.org/10.5281/zenodo.2586989). RNA-Seq reads were considered mapped 510 if the alignment shows at least 95\% similarity and covers $90 \%$ of the read length. 511 Splice sites were considered valid if they are spanned by at least three reads and 512 show a coverage drop of $20 \%$ when moving from an exon into an intron sequence. 513 Summaries of the RNA-Seq read coverage depth at splice sites in animals ${ }^{57}$ and 514 fungi ${ }^{58}$ have been made available as part of this study.

515 RNA-Seq read mappings with STAR v2.5.1b and HiSat2 v.2.1.0 were compared 516 based on a gold standard generated by exonerate, because a previous report ${ }^{59}$ 517 indicated a superiority of STAR. STAR parameters were set as described above and 518 HiSat2 was applied with default parameters. All transcripts with non-canonical splice 519 sites in $A$. thaliana and Oryza sativa were considered. When investigating the 520 alignment of RNA-Seq reads over non-canonical splice sites, we observed a high 521 accuracy for both mappers without a clear difference between them. Previously 522 described scripts ${ }^{10}$ were adjusted for this analysis and updated versions are 523 available on github (https://doi.org/10.5281/zenodo.2586989). The distribution of 524 genome sizes was analysed using the Python package dabest ${ }^{60}$. Sequence logos for 525 the analysed splice sites were designed at http://weblogo.berkeley.edu/logo.cgi ${ }^{61}$.

\section{Calculation of the splice site diversity}

528 A custom Python script (splice_site_divergence_check.py) was applied to calculate 529 the Shannon diversity index $\left(\mathrm{H}^{\prime}\right){ }^{62}$ of all splice site combinations in fungi, animals 
530 and plants (https://doi.org/10.5281/zenodo.2586989). To determine the significance

531 of the obtained results, a Kruskal-Wallis test ${ }^{63}$ was calculated using the Python

532 package scipy ${ }^{64}$. Further, the interquartile range of all distributions was examined.

533

534 Investigation of a common non-canonical splice site in fungal genome 535 sequences

536 A Mann-Whitney $\cup$ Test implemented in the Python package scipy was performed to 537 analyse differences in the number of minor non-canonical splice site combinations. 538 The observed distributions were visualized in a boxplot 539 (https://doi.org/10.5281/zenodo.2586989) constructed with the Python package plotly 54065 (ss_combination_frequency_boxplot.py).

541

\section{Detection of spliceosomal components}

543 A potential U1 snRNA of Pan troglodytes (obtained from the NCBI; 544 GeneID:112207549) was subjected to BLASTn v.2.8.1 ${ }^{66}$ against the genome 545 sequences of selected species. Hits with a score above 100, with at least $80 \%$ 546 similarity and with the conserved sequence at the $5^{\prime}$ end of the snRNA ${ }^{67}$ were 547 investigated, as these sequences are potential U1 snRNAs. The obtained sequences 548 were compared and small nucleotide variants were detected.

549 To assess possible duplications of spliceosomal components, bait sequences from 550 various species were collected for central proteins in the spliceosome including pre551 mRNA-processing factor 8 (PRP8), U1 small nuclear ribonucleoprotein C, U4/U6 552 small nuclear ribonucleoprotein Prp3, U4/U6.U5 small nuclear ribonucleoprotein 27 $553 \mathrm{kDa}$ protein and U5 small nuclear ribonucleoprotein. Putative homologues in all 554 animal species were detected based on Python scripts ${ }^{68}$ and subjected to the 555 construction of phylogenetic trees as described previously ${ }^{69}$.

556 Genome sequences were systematically screened for U12 spliceosome hints via 557 Infernal (cmscan) v1.1.2 ${ }^{70}$ with Rfam13 ${ }^{71}$. U4atac, U6atac, U11, and U12 were 558 considered as indications for the presence of the minor U12 spliceosome in the 559 respective species. Due to high computational costs only a random subset of all 560 animal sequences was analysed. 
562 Correlation between the GC content of the genome and the GC content of the 563 splice sites

564 The Pearson correlation coefficient between the GC content of the genome 565 sequence of each species and the GC content of the respective splice site 566 combination was calculated using the Python package scipy. Splice site 567 combinations were weighted with the number of occurrences for assessment of the 568 GC content. Finally, the correlation coefficient and the p-value were determined. For 569 better visualization, a scatter plot was constructed with the Python package plotly ${ }^{65}$.

570

\section{Phylogeny of non-canonical splice sites}

572 All $A$. thaliana transcripts with non-canonical splice sites were subjected to BLASTn 573 v.2.8.1 searches against the transcript sequences of all other plant species 574 previously studied ${ }^{10}$. The best hit per species was selected for an alignment against 575 the respective genomic region with exonerate ${ }^{72}$. Next, splice site combinations were 576 extracted and aligned. This alignment utilized MAFFT $\vee 7{ }^{73}$ by representing different 577 splice site combinations as amino acids. Finally, splice site combinations aligned with 578 the non-canonical splice site combinations of $A$. thaliana were analysed 579 (https://doi.org/10.5281/zenodo.2586989).

580 All transcripts of the fungus Armillaria gallica with non-canonical splice sites were 581 searched as translated peptide sequences against all other fungal peptide sequence 582 data sets via BLASTp ${ }^{66}$. Cases with more than 10 best hits with non-canonical splice 583 site combinations in other species were subjected to the construction of phylogenetic 584 trees for manual inspection. MAFFT v7 ${ }^{73}$ and FastTree v2 ${ }^{74}$ were used for the 585 alignment and tree construction.

586

\section{Usage of non-canonical splice sites}

588 Genes were classified based on the presence/absence of non-canonical splice 589 combinations into four groups: GT-AG, GC-AG, AT-AC, and minor non-canonical 590 splice site genes. When having different non-canonical splice sites, genes were 591 assigned into multiple groups. Next, the transcription of these genes was quantified 
592 based on RNA-Seq using featureCounts v1.5.0-p3 ${ }^{75}$ based on the RNA-Seq read 593 mapping generated with STAR v.2.5.1b. Multi-mapped reads were excluded from the 594 analysis and expression values were calculated at gene level (-t gene). Binning of the 595 genes was performed based on the fragments per kilobase transcript length per 596 million assigned fragments (FPKMs). Despite various shortcomings ${ }^{76}$, we consider 597 FPKMs to be acceptable for this analysis. Outlier genes with extremely high values 598 were excluded from this analysis and the visualization. Next, a cumulative sum of the 599 relative bin sizes was calculated. The aim was to compare the transcriptional activity 600 of genes with different splice site combinations i.e. to test whether non-canonical 601 splice site combinations are enriched in lowly transcribed genes.

602 Usage of splice sites was calculated per intron as previously described ${ }^{10}$. The 603 difference between both ends of an intron was calculated. The distribution of these 604 differences per splice site type were analysed.

605 Introns were grouped by their splice site combination. The average of both coverage 606 values of the directly flanking exon positions was calculated as estimate of the local 607 expression around a splice site combination. Next, the sequencing coverage of a 608 transcript was estimated by multiplying 200 bp (assuming 2x100 nt reads) with the 609 number of read counts per gene and normalization to the transcript length. The 610 difference between both values was calculated for each intron to assess its presence 611 in the major isoform.

\section{Genomic read mapping and variant calling}

614 Genomic sequencing reads were retrieved from the SRA via fastq-dump as 615 described above. BWA MEM v.0.7 ${ }^{77}$ was applied with the $-\mathrm{m}$ parameter for mapping 616 of the reads and GATK v.3.8 ${ }^{78,79}$ was used for the variant detection as described 617 previously ${ }^{80}$. The positions of variants were compared to the positions of splice sites 618 using compare_variation_rates.py (https://github.com/bpucker/ncss2018).

\section{Data Availability}

621 This work was based on publicly available data sets retrieved from the NCBI 622 (Supplementary Data S3, Supplementary Data S4) and the SRA (Supplementary 
623 Data S17, Supplementary Data S18). Python scripts and a detailed technical 624 description are available at github: https://github.com/bpucker/ncss2018. Data sets 625 with information about the coverage around splice sites in animals 626 (http://doi.org/10.4119/unibi/2934226) and fungi 627 (http://doi.org/10.4119/unibi/2934220) were made available as data publication at 628 Bielefeld University Library.

629

630

\section{Acknowledgments}

632 We thank members of Genetics and Genomics of Plants for discussion of preliminary 633 results. We are very grateful to Hanna Marie Schilbert, Janik Sielemann, and lain 634 Place for helpful comments on the manuscript. We acknowledge support for the 635 Article Processing Charge by the Deutsche Forschungsgemeinschaft and the Open 636 Access Publication Fund of Bielefeld University.

637

\section{Author Contributions Statement}

639 K.F. and B.P. designed the study, performed the experiments, analysed the data, 640 and wrote the manuscript. Both authors read and approved the final version of this 641 manuscript.

642

\section{Competing interests}

644 The authors declare no competing interests.

645

646

647

648

649

650

651 


\section{References}

653 1. Moore, Melissa J and Sharp, Phillip A. Site-specific modification of pre-mRNA: the

654 2'-hydroxyl groups at the splice sites. Science 256, 992-997 (1992).

655 2. Barbosa-Morais, Nuno L and Irimia, Manuel and Pan, Qun and Xiong, Hui $Y$ and

656 Gueroussov, Serge and Lee, Leo J and Slobodeniuc, Valentina and Kutter,

657 Claudia and Watt, Stephen and Çolak, Recep and others. The evolutionary

658 landscape of alternative splicing in vertebrate species. Science 338, 1587-1593

659 (2012).

660 3. Ben-Dov, Claudia and Hartmann, Britta and Lundgren, Josefin and Valcárcel,

661 Juan. Genome-wide analysis of alternative pre-mRNA splicing. J. Biol. Chem. 283,

$662 \quad 1229-1233(2008)$.

663 4. Matlin, Arianne J and Clark, Francis and Smith, Christopher WJ. Understanding

664 alternative splicing: towards a cellular code. Nat. Rev. Mol. Cell Biol. 6, 386 (2005).

665 5. Sibley, Christopher R and Blazquez, Lorea and Ule, Jernej. Lessons from non-

666 canonical splicing. Nat. Rev. Genet. 17, 407 (2016).

667 6. Maniatis, Tom and Tasic, Bosiljka. Alternative pre-mRNA splicing and proteome

668 expansion in metazoans. Nature 418, 236 (2002).

669 7. Xue, Min and Chen, Bing and Ye, Qingqing and Shao, Jingru and Lyu, Zhangxia

670 and Wen, Jianfan. Sense-antisense gene overlap causes evolutionary retention of

671 the few introns in Giardia genome and the implications. bioRxiv (2018).

672 8. Chorev, Michal and Carmel, Liran. The function of introns. Front. Genet. 3, (2012).

673 9. Burset, M and Seledtsov, IA and Solovyev, VV. Analysis of canonical and non-

674 canonical splice sites in mammalian genomes. Nucleic Acids Res. 28, 4364-4375

675 (2000). 
676 10. Pucker, Boas and Brockington, Samuel F. Genome-wide analyses supported 677 by RNA-Seq reveal non-canonical splice sites in plant genomes. BMC Genomics $678 \quad 19,980(2018)$.

679 11. Bon, Elisabeth and Casaregola, Serge and Blandin, Gaëlle and Llorente, 680 Bertrand and Neuvéglise, Cécile and Munsterkotter, Martin and Guldener, Ulrich 681 and Mewes, Hans-Werner and Helden, Jacques Van and Dujon, Bernard and 682 others. Molecular evolution of eukaryotic genomes: hemiascomycetous yeast 683 spliceosomal introns. Nucleic Acids Res. 31, 1121-1135 (2003).

684 12. Logsdon, John M. The recent origins of spliceosomal introns revisited. Curr. 685 Opin. Genet. Dev. 8, 637-648 (1998).

686 13. Burge, Chris and Karlin, Samuel. Prediction of complete gene structures in 687 human genomic DNA1. J. Mol. Biol. 268, 78-94 (1997).

688 14. Stanke, Mario and Waack, Stephan. Gene prediction with a hidden Markov 689 model and a new intron submodel. Bioinformatics 19, ii215-ii225 (2003).

690 15. Davis, Carrie A and Grate, Leslie and Spingola, Marc and Ares Jr, Manuel. 691 Test of intron predictions reveals novel splice sites, alternatively spliced mRNAs 692 and new introns in meiotically regulated genes of yeast. Nucleic Acids Res. 28, $693 \quad 1700-1706(2000)$.

694 16. Wahl, Markus $C$ and Will, Cindy L and Lührmann, Reinhard. The spliceosome: 695 design principles of a dynamic RNP machine. Cell 136, 701-718 (2009).

696 17. Sharp, Phillip A and Burge, Christopher B. Classification of introns: U2-type or 697 U12-type. Cell 91, 875-879 (1997).

698 18. Hall, Stephen $L$ and Padgett, Richard A. Requirement of U12 snRNA for in 699 vivo splicing of a minor class of eukaryotic nuclear pre-mRNA introns. Science $700 \quad 271,1716-1718$ (1996). 
701 19. Turunen, Janne $\mathrm{J}$ and Niemelä, Elina $\mathrm{H}$ and Verma, Bhupendra and Frilander,

702 Mikko J. The significant other: splicing by the minor spliceosome. Wiley Interdiscip.

703 Rev. RNA 4, 61-76 (2013).

704 20. Dietrich, Rosemary C and Incorvaia, Robert and Padgett, Richard A. Terminal

705 intron dinucleotide sequences do not distinguish between U2-and U12-dependent

706 introns. Mol. Cell 1, 151-160 (1997).

707 21. Wilkinson, Max E and Fica, Sebastian M and Galej, Wojciech P and Norman,

708 Christine M and Newman, Andrew J and Nagai, Kiyoshi. Postcatalytic spliceosome

709 structure reveals mechanism of 3'-splice site selection. Science 358, 1283-1288

710 (2017).

711 22. Burge, Christopher B and Tuschl, Thomas and Sharp, Phillip A. Splicing of

712 precursors to mRNAs by the spliceosomes. Cold Spring Harb. Monogr. Ser. 37,

$713 \quad 525-560$ (1999).

714 23. Roca, Xavier and Krainer, Adrian R and Eperon, lan C. Pick one, but be quick:

715 5' splice sites and the problems of too many choices. Genes Dev. 27, 129-144

716 (2013).

717 24. Shi, Yigong. The spliceosome: a protein-directed metalloribozyme. J. Mol. Biol.

$718 \quad 429,2640-2653$ (2017).

719 25. Kupfer, Doris M and Drabenstot, Scott D and Buchanan, Kent L and Lai,

720 Hongshing and Zhu, Hua and Dyer, David W and Roe, Bruce A and Murphy,

721 Juneann W. Introns and splicing elements of five diverse fungi. Eukaryot. Cell 3,

$722 \quad 1088-1100$ (2004).

723 26. Kitamura-Abe, Sumie and Itoh, Hitomi and Washio, Takanori and Tsutsumi,

724 Akihiro and Tomita, Masaru. Characterization of the splice sites in GT-AG and

725 GC-AG introns in higher eukaryotes using full-length cDNAs. J. Bioinform.

726 Comput. Biol. 2, 309-331 (2004). 
727 27. Michael, Deutsch and Manyuan, Long. Intron-exon structures of eukaryotic

728 model organisms. Nucleic Acids Res. 27, 3219-3228 (1999).

729 28. Modrek, Barmak and Resch, Alissa and Grasso, Catherine and Lee,

730 Christopher. Genome-wide detection of alternative splicing in expressed

731 sequences of human genes. Nucleic Acids Res. 29, 2850-2859 (2001).

732 29. Pucker, Boas and Holtgräwe, Daniela and Weisshaar, Bernd. Consideration of 733 non-canonical splice sites improves gene prediction on the Arabidopsis thaliana

734 Niederzenz-1 genome sequence. BMC Res. Notes 10, 667 (2017).

735 30. Sparks, Michael E and Brendel, Volker. Incorporation of splice site probability

736 models for non-canonical introns improves gene structure prediction in plants.

737 Bioinformatics 21, iii20-iii30 (2005).

738 31. Dubrovina, AS and Kiselev, KV and Zhuravlev, Yu N. The role of canonical 739 and noncanonical pre-mRNA splicing in plant stress responses. BioMed Res. Int.

740 2013, (2013).

741 32. Alexandrov, Nickolai $\mathrm{N}$ and Troukhan, Maxim $\mathrm{E}$ and Brover, Vyacheslav $\mathrm{V}$ and 742 Tatarinova, Tatiana and Flavell, Richard B and Feldmann, Kenneth A. Features of 743 Arabidopsis genes and genome discovered using full-length cDNAs. Plant Mol.

744 Biol. 60, 69-85 (2006).

745 33. Niu, Xiangli and Luo, Di and Gao, Shaopei and Ren, Guangjun and Chang, 746 Lijuan and Zhou, Yuke and Luo, Xiaoli and Li, Yuxiang and Hou, Pei and Tang,

747 Wei and others. A conserved unusual posttranscriptional processing mediated by

748 short, direct repeated (SDR) sequences in plants. J. Genet. Genomics 37, 85-99

749 (2010).

750 34. Erkelenz, Steffen and Theiss, Stephan and Kaisers, Wolfgang and Ptok, 751 Johannes and Walotka, Lara and Müller, Lisa and Hillebrand, Frank and Brillen, 752 Anna-Lena and Sladek, Michael and Schaal, Heiner. Ranking noncanonical 5' 
753 splice site usage by genome-wide RNA-seq analysis and splicing reporter assays.

754 Genome Res. 28, 1826-1840 (2018).

755 35. Grützmann, Konrad and Szafranski, Karol and Pohl, Martin and Voigt, Kerstin

756 and Petzold, Andreas and Schuster, Stefan. Fungal alternative splicing is

757 associated with multicellular complexity and virulence: a genome-wide multi-

758 species study. DNA Res. 21, 27-39 (2013).

759 36. Parada, G. E., Munita, R., Cerda, C. A. \& Gysling, K. A comprehensive survey 760 of non-canonical splice sites in the human transcriptome. Nucleic Acids Res. 42 , $761 \quad$ 10564-10578 (2014).

762 37. Keren, H., Lev-Maor, G. \& Ast, G. Alternative splicing and evolution:

763 diversification, exon definition and function. Nat. Rev. Genet. 11, 345 (2010).

764 38. Jackson, I. J. A reappraisal of non-consensus mRNA splice sites. Nucleic

$765 \quad$ Acids Res. 19, 3795 (1991).

766 39. Robertson, Hugh M. Non-canonical GA and GG 5'Intron Donor Splice Sites

767 Are Common in the Copepod Eurytemora affinis. G3 Genes Genomes Genet. g3$768300189(2017)$.

769 40. Lee, C. E. Evolutionary mechanisms of habitat invasions, using the copepod

770 Eurytemora affinis as a model system. Evol. Appl. 9, 248-270 (2016).

771 41. Seo, H.-C. et al. Miniature genome in the marine chordate Oikopleura dioica.

772 Science 294, 2506-2506 (2001).

773 42. Brackenridge, Simon and Wilkie, Andrew OM and Screaton, Gavin R. Efficient 774 use of a 'dead-end'GA 5" splice site in the human fibroblast growth factor receptor 775 genes'. EMBO J. 22, 1620-1631 (2003).

776 43. Mount, S. M. A catalogue of splice junction sequences. Nucleic Acids Res. 10, $777 \quad$ 459-472 (1982). 
778 44. Tyler, Brett M. Phytophthora sojae: root rot pathogen of soybean and model

779 oomycete. Mol. Plant Pathol. 8, 1-8 (2007).

780 45. Förster, Helga and Coffey, Michael D and Elwood, Hille and Sogin, Mitchell L.

781 Sequence analysis of the small subunit ribosomal RNAs of three zoosporic fungi

782 and implications for fungal evolution. Mycologia 306-312 (1990).

783 46. Shen, Danyu and Ye, Wenwu and Dong, Suomeng and Wang, Yuanchao and

784 Dou, Daolong. Characterization of intronic structures and alternative splicing in

785 Phytophthora sojae by comparative analysis of expressed sequence tags and

786 genomic sequences. Can. J. Microbiol. 57, 84-90 (2011).

787 47. Garalde, D. R. et al. Highly parallel direct RNA sequencing on an array of

788 nanopores. Nat. Methods 15, 201 (2018).

789 48. Aebi, $\mathrm{M}$ and Hornig, $\mathrm{H}$ and Padgett, RA and Reiser, $\mathrm{J}$ and Weissmann, $\mathrm{C}$.

790 Sequence requirements for splicing of higher eukaryotic nuclear pre-mRNA. Cell

$791 \quad 47,555-565$ (1986).

792 49. Talerico, MELISSA and Berget, SUSAN M. Effect of 5'splice site mutations on

793 splicing of the preceding intron. Mol. Cell. Biol. 10, 6299-6305 (1990).

794 50. Berget, Susan M. Exon recognition in vertebrate splicing. J. Biol. Chem. 270,

$795 \quad 2411-2414$ (1995).

796 51. Kuhn, J. M., Breton, G. \& Schroeder, J. I. mRNA metabolism of flowering-time

797 regulators in wild-type Arabidopsis revealed by a nuclear cap binding protein

798 mutant, abh1. Plant J. 50, 1049-1062 (2007).

799 52. Donaldson, M. E. \& Saville, B. J. Natural antisense transcripts in fungi: Natural

800 antisense transcripts in fungi. Mol. Microbiol. 85, 405-417 (2012).

801 53. Simão, Felipe $A$ and Waterhouse, Robert $M$ and loannidis, Panagiotis and

802 Kriventseva, Evgenia V and Zdobnov, Evgeny M. BUSCO: assessing genome 
803 assembly and annotation completeness with single-copy orthologs. Bioinformatics

$804 \quad 31,3210-3212$ (2015).

805 54. Kriventseva, Evgenia V and Tegenfeldt, Fredrik and Petty, Tom J and

806 Waterhouse, Robert M and Simao, Felipe A and Pozdnyakov, Igor A and

807 Ioannidis, Panagiotis and Zdobnov, Evgeny M. OrthoDB v8: update of the

808 hierarchical catalog of orthologs and the underlying free software. Nucleic Acids

809 Res. 43, D250-D256 (2014).

810 55. Leinonen, Rasko and Sugawara, Hideaki and Shumway, Martin and

811 International Nucleotide Sequence Database Collaboration. The sequence read

812 archive. Nucleic Acids Res. 39, D19-D21 (2010).

813 56. Dobin, Alexander and Davis, Carrie A and Schlesinger, Felix and Drenkow,

814 Jorg and Zaleski, Chris and Jha, Sonali and Batut, Philippe and Chaisson, Mark

815 and Gingeras, Thomas R. STAR: ultrafast universal RNA-seq aligner.

816 Bioinformatics 29, 15-21 (2013).

817 57. Pucker, Boas and Frey, Katharina. RNA-Seq read coverage depth of splice 818 sites in animals. (2019).

819 58. Pucker, Boas and Frey, Katharina. RNA-Seq read coverage depth of splice 820 sites in fungi. (2019).

821 59. Dobin, Alexander and Gingeras, Thomas R. Comment on "TopHat2: accurate 822 alignment of transcriptomes in the presence of insertions, deletions and gene 823 fusions" by Kim et al.

824 https://www.biorxiv.org/content/10.1101/000851v1.full?\%3Fcollection= (2013).

825 60. Ho, Joses and Tumkaya, Tayfun and Aryal, Sameer and Choi, Hyungwon and

826 Claridge-Chang, Adam. Moving beyond P values: Everyday data analysis with

827 estimation plots. bioRxiv 377978 (2018). 
828 61. Crooks, Gavin E and Hon, Gary and Chandonia, John-Marc and Brenner,

829 Steven E. WebLogo: a sequence logo generator. Genome Res. 14, 1188-1190

830 (2004).

831 62. Heip, Carlo. A new index measuring evenness. J. Mar. Biol. Assoc. U. K. 54,

$832 \quad 555-557$ (1974).

833 63. Breslow, Norman. A generalized Kruskal-Wallis test for comparing K samples 834 subject to unequal patterns of censorship. Biometrika 57, 579-594 (1970).

835 64. Eric Jones and Travis Oliphant and Pearu Peterson and others. SciPy: Open 836 source scientific tools for Python. (2001).

837 65. Plotly Technologies Inc. Collaborative data science. https://plot.ly (2015).

838 66. Altschul, Stephen F and Gish, Warren and Miller, Webb and Myers, Eugene W 839 and Lipman, David J. Basic local alignment search tool. J. Mol. Biol. 215, 403-410 840 (1990).

841 67. Stark, Holger and Dube, Prakash and Lührmann, Reinhard and Kastner, 842 Berthold. Arrangement of RNA and proteins in the spliceosomal U1 small nuclear 843 ribonucleoprotein particle. Nature 409, 539 (2001).

844 68. Yang, Y. et al. Dissecting Molecular Evolution in the Highly Diverse Plant 845 Clade Caryophyllales Using Transcriptome Sequencing. Mol. Biol. Evol. 32, 20018462014 (2015).

847 69. Schilbert, H. M., Pellegrinelli, V., Rodriguez-Cuenca, S., Vidal-Puig, A. \& 848 Pucker, B. Harnessing natural diversity to identify key amino acid residues in 849 prolidase. http://biorxiv.org/lookup/doi/10.1101/423475 (2018) doi:10.1101/423475.

850 70. Nawrocki, E. P. \& Eddy, S. R. Infernal 1.1: 100-fold faster RNA homology 851 searches. Bioinformatics 29, 2933-2935 (2013).

852 71. Kalvari, I. et al. Rfam 13.0: shifting to a genome-centric resource for non853 coding RNA families. Nucleic Acids Res. 46, D335-D342 (2018). 
854 72. Slater, Guy St C and Birney, Ewan. Automated generation of heuristics for 855 biological sequence comparison. BMC Bioinformatics 6, 31 (2005).

856 73. Katoh, Kazutaka and Standley, Daron M. MAFFT multiple sequence alignment 857 software version 7: improvements in performance and usability. Mol. Biol. Evol. 30, $858 \quad 772-780(2013)$.

859 74. Price, Morgan N and Dehal, Paramvir S and Arkin, Adam P. FastTree 2860 approximately maximum-likelihood trees for large alignments. PloS One 5, e9490 861 (2010).

862 75. Liao, Yang and Smyth, Gordon K and Shi, Wei. featureCounts: an efficient 863 general purpose program for assigning sequence reads to genomic features.

864 Bioinformatics 30, 923-930 (2013).

865 76. Conesa, Ana and Madrigal, Pedro and Tarazona, Sonia and Gomez-Cabrero, 866 David and Cervera, Alejandra and McPherson, Andrew and Szcześniak, Michall 867 Wojciech and Gaffney, Daniel J and Elo, Laura L and Zhang, Xuegong and others. 868 A survey of best practices for RNA-seq data analysis. Genome Biol. 17, 13 (2016). 869 77. Li, H. Aligning sequence reads, clone sequences and assembly contigs with 870 BWA-MEM. ArXiv Prepr. ArXiv13033997 (2013).

871 78. McKenna, A. et al. The Genome Analysis Toolkit: a MapReduce framework for 872 analyzing next-generation DNA sequencing data. Genome Res. 20, 1297-1303 873 (2010).

874 79. Van der Auwera, G. A. et al. From FastQ data to high-confidence variant calls: 875 the genome analysis toolkit best practices pipeline. Curr. Protoc. Bioinforma. 43, $876 \quad 11-10(2013)$.

877 80. Baasner, J.-S., Howard, D. \& Pucker, B. Influence of neighboring small 878 sequence variants on functional impact prediction. BioRxiv (2019). 


\section{Supplementary Data}

881 SupplementaryData S1. List of all possible splice site combinations in animal 882 species.

883 Supplementary Data S2. List of all possible splice site combinations in fungal 884 species.

885 Supplementary Data S3. List of genome sequences and annotations of the 886 investigated animal species.

887 Supplementary S4. List of genome sequences and annotations of the investigated 888 fungal species.

889 Supplementary Data S5. Distribution of genome sizes of all species.

890 Supplementary Data S6. Genome statistics concerning each analysed animal 891 species and status of U12 spliceosome.

892 Supplementary Data S7. Genome statistics concerning each analysed fungal species 893 and status of U12 spliceosome.

894 Supplementary Data S8. Distribution of canonical and non-canonical splice sites per 895 species in the animal kingdom.

896 Supplementary Data S9. Distribution of canonical and non-canonical splice sites per 897 species in the fungal kingdom.

898 Supplementary Data S10. Percentage of variants in canonical splice sites, major and 899 minor non-canonical splice sites.

900 Supplementary Data S11. Correlation between the GC content of the genome and 901 the GC content of the splice sites per kingdom.

902 Supplementary Data S12. Correlation between the size of the used RNA-Seq data 903 sets and the number of supported splice sites.

904 Supplementary Data S13. Proportion of genes with non-canonical splice sites in 905 dependence of the number of introns.

906 Supplementary Data S14. Usage of non-canonical splice sites in animals and fungi.

907 Supplementary Data S15. Non-canonical splice sites in BUSCOs and in all genes 908 were assessed per species in the animal kingdom.

909 Supplementary Data S16. Non-canonical splice sites in BUSCOs and in all genes 910 were assessed per species in the fungal kingdom.

911 Supplementary Data S17. List of Sequence Read Archive accession numbers of the 912 investigated animal RNA-Seq data sets.

913 Supplementary Data S18. List of Sequence Read Archive accession numbers of the 914 investigated fungal RNA-Seq data sets. 


\section{a 0.1}

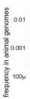

o.

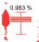

D.75e $>$ :
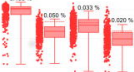

GCNG ATUAC GANG GT-TG GGAE

b

?. $5, \mathrm{nt}$

$\stackrel{0}{\circ}$

g

$+$

它

$\dot{5}$

党

ํㅗㄹ $104 x$

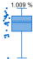

incos $\mathrm{x}$

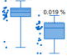

, $0010=$

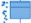

i-

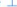

4. 31708

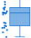

GE.AG CTAE AT-AC GA-AG GI-TG

C

政

จำ

要

in

要

$3.00 \%$

9
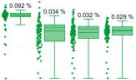

GCAG ATAC GT.TG GTHA GA.AG 


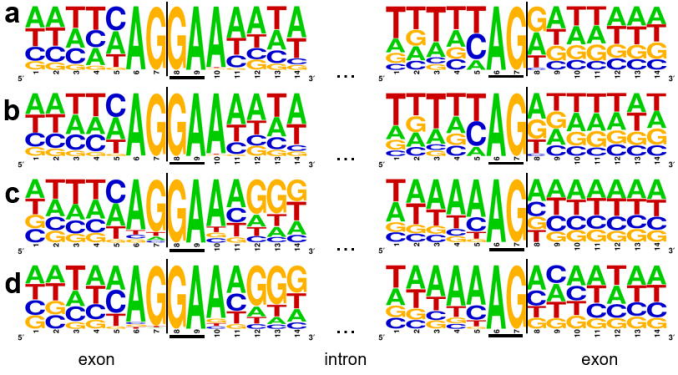




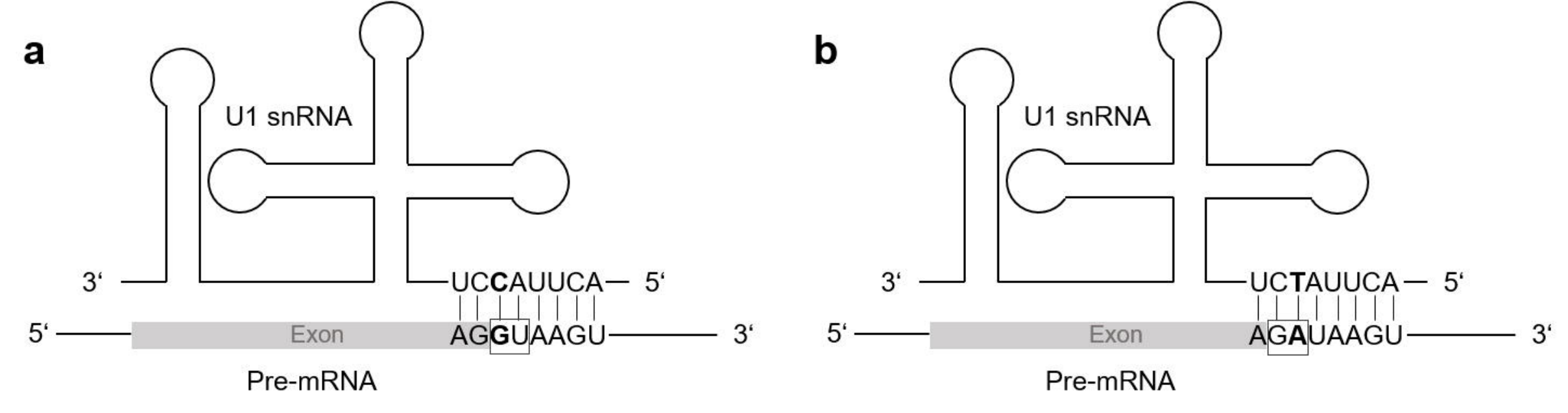




\section{$\square \mathrm{AT}-\mathrm{AC} \square \mathrm{CT}-\mathrm{AC}$}

280

ᄃ 260

을 240

. 220

है 200

ㅇ 180

品 160

क 140

응 120

कㅇ 100

¿० 80

80

Е
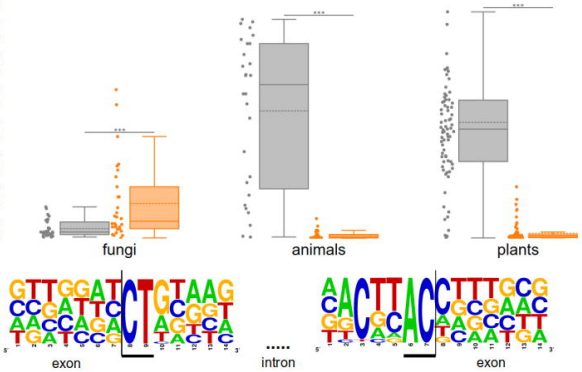

intron

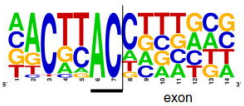



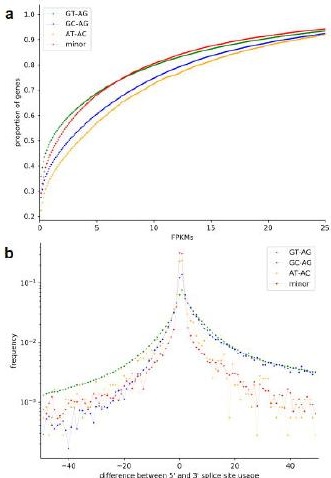